\title{
Rho-Kinase Inhibition Attenuates Acute Hypoxic Fetoplacental Vasoconstriction in the Rat
}

\author{
P. KAFKA ${ }^{1,2}$, O. VAJNEROVÁ ${ }^{1}$, J. HERGET $^{1}$, V. HAMPL $^{1}$ \\ ${ }^{1}$ Department of Physiology, Second Faculty of Medicine, Charles University, Prague, Czech \\ Republic, ${ }^{2}$ Department of Anaesthesiology and Intensive Care Medicine, Third Faculty of \\ Medicine, Charles University, Prague, Czech Republic
}

Received April 6, 2012

Accepted May 28, 2012

\begin{abstract}
Summary
The vessels on the fetal side of the placenta differ from most other vascular beds except the lungs in that they respond to acute hypoxia by vasoconstriction. An essential role of calcium influx in the mechanism of this hypoxic fetoplacental vasoconstriction (HFPV) has been shown previously. That finding does not, however, exclude the possible involvement of other mechanisms of vascular tone regulation. In this study we tested the hypothesis that Rho-kinase-mediated calcium sensitization is involved in HFPV. We used a model of isolated rat placenta dually perfused (from both the maternal and fetal side) with Krebs salt solution saturated with normoxic and hypoxic gas mixture respectively at constant flow rate. Rho-kinase pathway was inhibited by fasudil $(10 \mu \mathrm{M})$. We found that fasudil reduced basal normoxic fetoplacental vascular resistance and completely prevented HFPV. This suggests that the activity of Rho-kinase signaling pathway is essential for HFPV.
\end{abstract}

\section{Key words}

Hypoxic fetoplacental vasoconstriction - Calcium sensitization • Rho-kinase pathway $\bullet$ Placenta $\bullet$ Fasudil

\section{Corresponding author}

Petr Kafka, Department of Physiology, Second Faculty of Medicine, Charles University, Plzeňská 130/221, 15000 Prague 5, Czech Republic. Fax: +420257210 995. E-mail: petrkaf@yahoo.com

\section{Introduction}

Reduction of partial pressure of oxygen in the perfusate of the maternal lacunas results in reversible vasoconstriction in the fetal part of the placenta (Howard
1987). The mechanism of this hypoxic fetoplacental vasoconstriction (HFPV) includes hypoxic inhibition of voltage-gated potassium channels in the plasma membrane of fetoplacental vascular smooth muscle (Hampl et al. 2002). Consequent membrane depolarization opens L-type of voltage-gated calcium channels (Jakoubek et al. 2006) and thus enables $\mathrm{Ca}^{2+}$ influx into the cytoplasm. $\mathrm{Ca}^{2+}$ binds to calmodulin and activates myosin light chain kinase (MLCK), leading to phosphorylation of light chain of myosin (MLC) (Somlyo and Somlyo 2003).

The degree of phosphorylation of MLC generally determines the degree of smooth muscle contraction due to the activation of the myosin ATPase. At the same time, phosphorylated MLC is dephosphorylated by MLC phosphatase (MLCP). Thus the balance between activities of MLCK (contraction) and MLCP (relaxation) regulates smooth muscle tone (Somlyo and Somlyo 2003). However, using $\mathrm{Ca}^{2+}$ indicators to measure intracellular $\mathrm{Ca}^{2+}$, it has been revealed that the extent of MLC phosphorylation and sustained smooth muscle contraction induced by agonist stimulation is higher than that caused by an increase in the $\mathrm{Ca}^{2+}$ concentration. This so-called $\mathrm{Ca}^{2+}$ sensitization (Somlyo and Somlyo 2003) is mediated by the signaling pathway that involves GTPase Rho and Rho-associated kinase (Rho-kinase), which is thought to act by inhibiting of the MLCP activity (for review see Fukata et al. 2001). Thus the inhibition of Rho-kinase signaling pathway desinhibits MLCP, leads to MLC dephosphorylation and therefore to smooth muscle relaxation. This assumption was proved by several authors (Wang et al. 2001, Robertson et al. 2000) in acute hypoxia-induced 
vasoconstriction of pulmonary vessels. Also, Fagan et al. (2004) demonstrated a significant attenuation of the hypoxic vasopressor response of isolated mouse lungs after the treatment with Y-27632, a Rho-kinase inhibitor. Pulmonary and placental vascular beds play equivalent role in oxygenation of the adult and fetal blood, respectively. For this functional similarity of lung and placental circulation we hypothesize similar mechanisms in the hypoxic vasopressor responses of both beds. In the present study we tested the hypothesis that Rho-kinase inhibition reduces both the basal vascular resistance and the vasoconstrictor response to hypoxia in fetal side of the placenta.
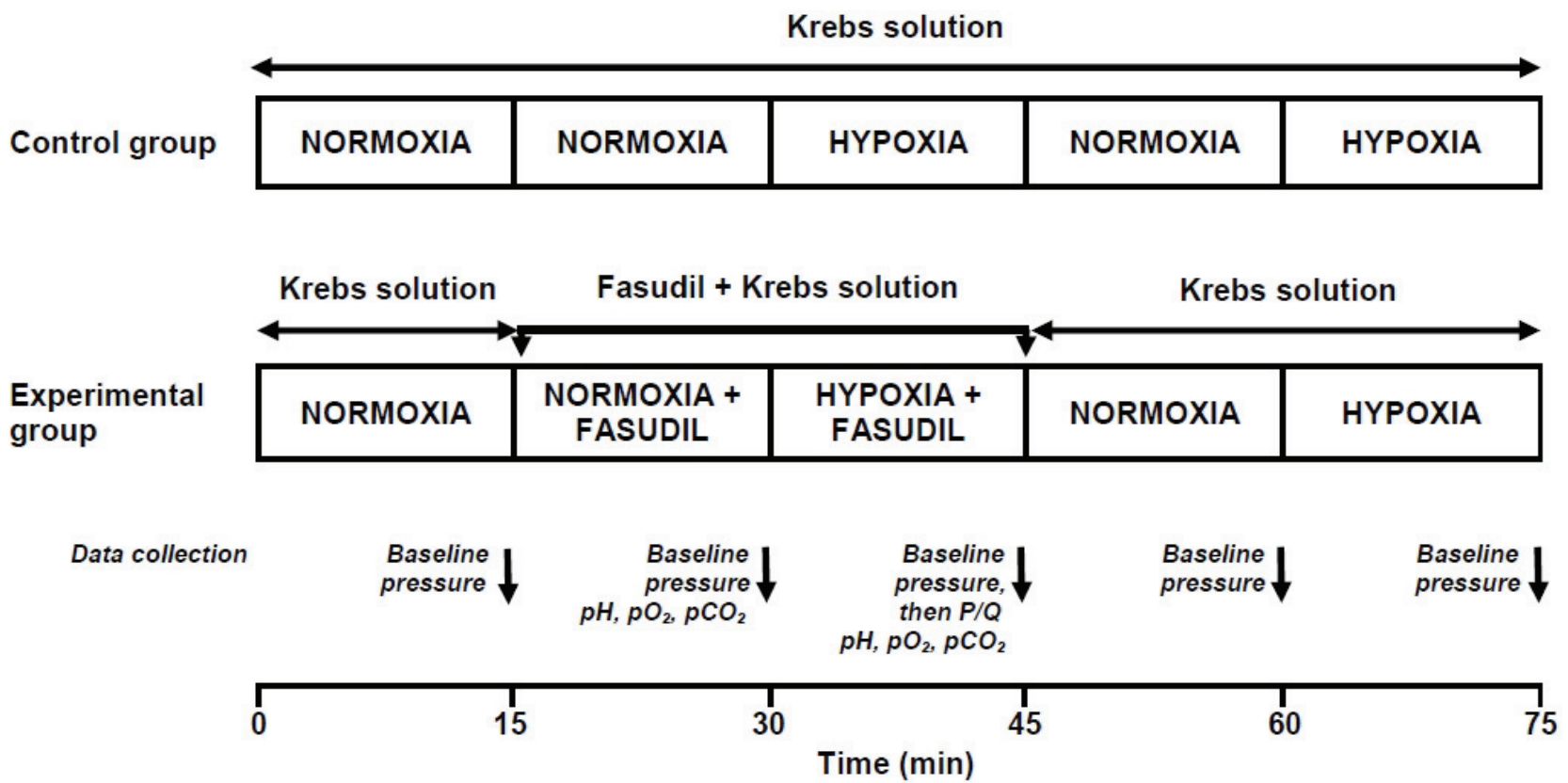

Fig 1. Experimental protocol. In the experimental group, the perfusate containing fasudil was replaced with a fresh one without fasudil after the $P / Q$ measurement.

\section{Methods}

\section{Animals and chemicals}

All animal manipulations and experimental protocol were in accordance with the European Guidelines on Laboratory Animal Care and were approved by the Animal Care Committee of the Second Faculty of Medicine (Charles University in Prague). Pregnant Wistar rats (Biotest, Konárovice, Czech Republic) weighing 380-540 g were used.

Rho-kinase inhibitor fasudil was purchased from LC Laboratories (Woburn, Massachusetts, USA). All other chemicals were obtained from Sigma-Aldrich (Prague, Czech Republic).

\section{Rat placenta isolation and perfusion}

The model of isolated, dually perfused rat placenta was prepared as described previously (Štulc and Štulcová 1986). One day before the expected date of delivery (day 20 of gravidity) each animal was anaesthetized with Thiopental (Valeant, Prague, Czech Republic, $50 \mathrm{mg} / \mathrm{kg}$ i.p.) and placed in a bath of Ringer's solution kept at $37^{\circ} \mathrm{C}$. The abominal wall was opened by a midline incision. One horn of the uterus was exposed and submerged in the heated Ringer's solution. A catheter was inserted into the uterine artery leading to the selected placenta and maternal side of the placenta was then perfused with Krebs solution $\left(37{ }^{\circ} \mathrm{C}\right)$ equilibrated with a normoxic gas mixture (bubbling with $21 \% \mathrm{O}_{2}, 5 \% \mathrm{CO}_{2}$ and $74 \% \mathrm{~N}_{2}$ in a reservoir). Flow rate was gradually increased to $1 \mathrm{ml} / \mathrm{min}$ and then kept constant. The uterine vein was ligated behind the selected placenta and carefully cut so that the perfusate could freely leave the placenta. The uterus was then opened and the fetuses were exposed. The umbilical artery of the selected placenta was catheterized to allow perfusion of the fetal side of the placenta (using the same perfusate as maternal side from a common reservoir) at $1 \mathrm{ml} / \mathrm{min}$. The umbilical vein was catheterized in a similar manner and was kept open at the level of placenta to allow free outflow of the perfusate. It 
was used for taking samples to measure outflow $\mathrm{pH}, \mathrm{pO}_{2}$ and $\mathrm{pCO}_{2}$ (ABL 5, Radiometer, Copenhagen, Denmark). All fetuses were then sacrificed by Thiopental overdose. Perfusion pressure on both sides of placenta (fetal and maternal) and flow were monitored by a PowerLab data acquisition system (ADInstruments, Spechbach, Germany). At the end of each experiment the mothers, fetuses and placentas were weighted and the weights of perfused and nonperfused placentas were compared.

\section{Experimental protocol}

The rats were randomly divided into two groups: control $(n=11)$ and experimental $(n=11)$ treated with fasudil (10 $\mu \mathrm{M}$ perfusate concentration). After the perfusion of both sides of the placenta from a common reservoir had been established, the experimental protocol was carried out (Fig. 1). Hypoxic vasoconstrictor responses were elicited by exchange of the gas mixture bubbling the reservoir to $95 \% \mathrm{~N}_{2}+5 \% \mathrm{CO}_{2}$ for $15 \mathrm{~min}$. The relationship between perfusion pressure and flow ( $\mathrm{P} / \mathrm{Q}$ ramp) was measured at the end of the first hypoxic challenge. To do so, the flow rate on the placental side was continuously increased in a ramp fashion from 0 to $2 \mathrm{ml} / \mathrm{min}$ over a $2 \mathrm{~min}$ period. To assess the reversibility of the fasudil effect, the perfusate was replaced with a fresh one without fasudil after the $\mathrm{P} / \mathrm{Q}$ ramp measurement in the experimental group.

\section{Statistical analysis}

Data are reported as means \pm SEM and were analyzed using StatView 5 software (SAS Institute, Cary, NC, USA). The differences between the groups were analyzed using unpaired $t$-test. The $\mathrm{P} / \mathrm{Q}$ ramp data were analyzed using linear regression and the differences in their parameters were compared between the groups using unpaired $t$-test. Differences were considered statistically significant when $\mathrm{P}<0.05$.

\section{Results}

Control and experimental groups did not differ in maternal body weights, weights of placentas and weights of fetuses (Table 1). To exclude placental edema due to perfusion we compared weights of perfused placentas (at the end of experiment) and weights of other non-perfused placentas (which were not part of our experiment) in both groups, and found no significant difference (control group $\mathrm{P}=0.16$, experimental group $\mathrm{P}=0.26$ ) (Table 1).

Characteristics of the fetoplacental effluent perfusate are given in Table 2. The drop in fetal effluent $\mathrm{pO}_{2}$ induced by acute hypoxic challenge (by $48 \pm 11$ $\mathrm{mmHg}$ in control group and $43 \pm 5$ in experimental group, $\mathrm{P}=0.72$ ) was unaffected by fasudil, the same was true for $\mathrm{pH}$ (the drop by $0.05 \pm 0.18 \mathrm{mmHg}$ in control group and $0.04 \pm 0.18 \mathrm{mmHg}$ in experimental group, $\mathrm{P}=0.24$ ).

Table 1. Maternal, placental and fetal weights.

\begin{tabular}{lcccc}
\hline Group & $\begin{array}{c}\text { Maternal body } \\
\text { weight }(\mathbf{g})\end{array}$ & $\begin{array}{c}\text { Weight of placentas } \\
\text { used for perfusion } \\
(\mathbf{m g})\end{array}$ & $\begin{array}{c}\text { Weight of other } \\
\text { placentas not used } \\
\text { in experiment (mg) }\end{array}$ & Fetal weight (g) \\
\hline $\begin{array}{l}\text { Control } \\
\text { Experimental }\end{array}$ & $438 \pm 12$ & $580 \pm 22$ & $591 \pm 19$ & $4.782 \pm 0.613$ \\
$\begin{array}{l}\text { P values (control } \\
\text { vs. experimental) }\end{array}$ & $440 \pm 12$ & $645 \pm 45$ & $668 \pm 33$ & $5.718 \pm 0.158$ \\
\hline
\end{tabular}

Table 2. Fetal effluent perfusate characteristics.

\begin{tabular}{lcccccc}
\hline \multirow{3}{*}{ Group } & \multicolumn{3}{c}{ Normoxia } & \multicolumn{3}{c}{ Hypoxia } \\
\cline { 2 - 7 } & $\mathbf{p H}$ & $\begin{array}{c}\mathbf{p O} \\
\mathbf{m m H g})\end{array}$ & $\begin{array}{c}\mathbf{p C O} \\
(\mathbf{m m H g})\end{array}$ & $\mathbf{p H}$ & $\begin{array}{c}\mathbf{p O}_{\mathbf{2}} \\
\mathbf{( m m H g )}\end{array}$ & $\begin{array}{c}\mathbf{p C O} \\
(\mathbf{m m H g})\end{array}$ \\
\hline $\begin{array}{l}\text { Control } \\
\text { Experimental }\end{array}$ & $7.28 \pm 0.02$ & $93 \pm 6$ & $32 \pm 1$ & $7.23 \pm 0.03$ & $46 \pm 5^{*}$ & $36 \pm 1^{*}$ \\
\hline
\end{tabular}

\footnotetext{
${ }^{*} \mathrm{P}<0.05$ vs. control group in normoxia. ${ }^{+} \mathrm{P}<0.01$ vs. experimental group in normoxia.
} 


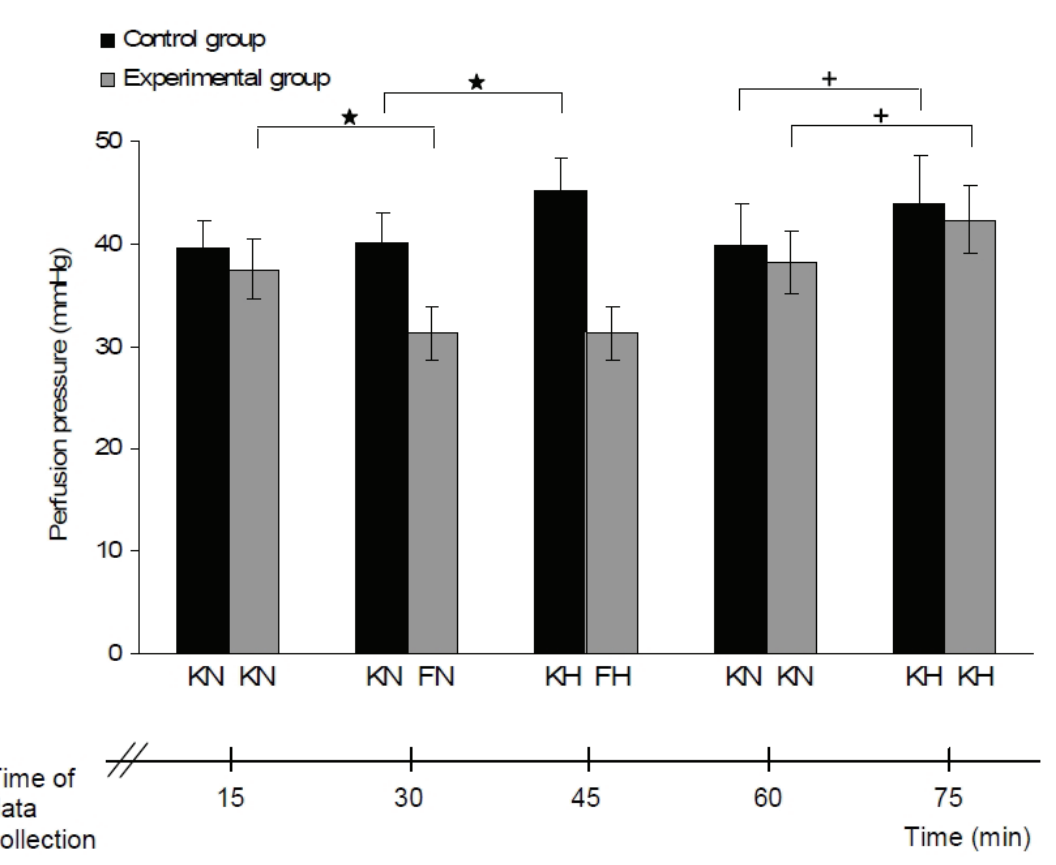

Fig. 2. Fetoplacental perfusion pressure at baseline perfusion flow. Perfusion pressure did not differ between the groups at the beginning of the experiment $(P=0.62)$. Fasudil caused a reduction of perfusion pressure in normoxia. Acute hypoxia induced HFPV in the control group $(P<0.01)$, but not in the fasudil-treated group. After the fasudil-containing perfusate was replaced with a fresh one without fasudil in the experimental group, HFPV of a similar magnitude was present in both groups. Perfusate specification: KN, Krebs solution under normoxia (gassed with a mixture of $21 \% \mathrm{O}_{2}, 5 \% \mathrm{CO}_{2}$ and $74 \%$ $\mathrm{N}_{2}$ ); $\mathrm{FN}$, Krebs solution with fasudil under normoxia; $\mathrm{KH}$, Krebs solution under hypoxia (gassed with a mixture of $95 \%$ $\mathrm{N}_{2}+5 \% \mathrm{CO}_{2}$ ); $\mathrm{FH}$, Krebs solution with fasudil under hypoxia. ${ }^{*} \mathrm{P}<0.01$, ${ }^{+} \mathrm{P}<0.05$.

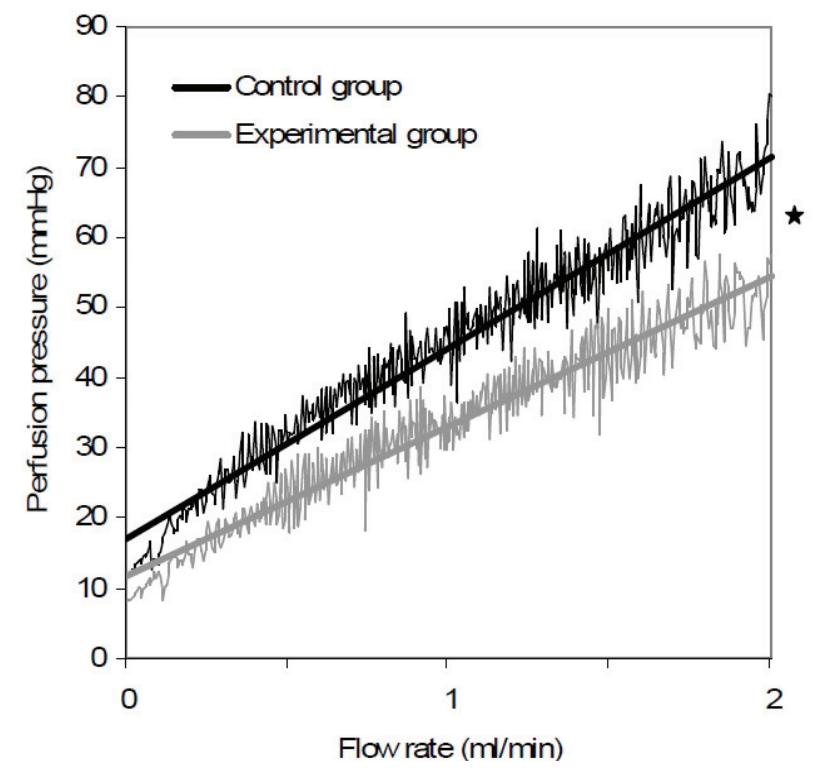

Fig. 3. Relationship between increasing fetoplacental perfusion pressure in response to continuously rising flow rate of perfusate (P/Q) during acute hypoxic challenge. Composite tracings from 11 placentas in each group and their linear regressions are shown. The slope of the $P / Q$ regression was significantly decreased in experimental group (perfused with fasudil) in comparison to control group ( $0.098 \pm 0.007$ vs. $0.125 \pm 0.009$, $\left.{ }^{*} \mathrm{P}<0.05\right)$. Extrapolated pressure axis intercept did not differ between the groups $(P=0.13)$.

Fetoplacental perfusion pressures at basic perfusion flow rate are shown in Fig. 2. The two groups did not differ in perfusion pressure before the fasudil addition. Fasudil caused a significant decrease of perfusion pressure in normoxia and completely prevented any hypoxic vasoconstrictor response (which was significant in the control group). Inhibition of HFPV by fasudil was completely reversible by replacing the fasudil-containing perfusate with a fresh Krebs solution without fasudil, which also normalized the baseline perfusion pressure.

The inhibition of HFPV by fasudil was confirmed by the $\mathrm{P} / \mathrm{Q}$ ramp data (measured during the hypoxic perfusion) (Fig. 3). The data were fitted into a linear regression model $\left(\mathrm{R}^{2}>0.95\right.$ in controls and $>0.94$ in the experimental group). The slope of the linear regression of the $\mathrm{P} / \mathrm{Q}$ data was significantly lower in the experimental than in the control group $(0.098 \pm 0.007$ vs. $0.125 \pm 0.009, \mathrm{P}<0.05)$. The intercept with pressure axis did not differ significantly between the groups.

\section{Discussion}

A previous report from our laboratory showed that even a very low dose of nifedipine $\left(10^{-9} \mathrm{M}\right)$, a selective inhibitor of the voltage-gated $\mathrm{Ca}^{2+}$ channels of the plasmalemma (L-type) completely abolished HFPV (Jakoubek et al. 2006). This implied an essential role of these channels in the HFPV mechanism. However, it is known from other vascular beds (Leach et al. 1994) that MLC phosphorylation achieved by other mechanisms may be needed to sustain vasoconstriction first initiated by $\mathrm{Ca}^{2+}$ influx. The main known mechanism of sustained MLC phosphorylation is the inactivation of MLCP through the Rho-kinase system (Sylvester et al. 2012). The present study does, indeed, show that the activity of 
Rho-kinase is also a prerequisite for HFPV. It should be mentioned that the two studies, on which we base this conclusion, were performed on different species. Even though we cannot exclude the possibility that L-type channels are more important in human placenta (Jakoubek et al. 2006) while $\mathrm{Ca}^{2+}$ sensitization prevails in the HFPV mechanism in the rat placenta (present study), we consider such a species difference unlikely. Most likely, both $\mathrm{Ca}^{2+}$ influx through L-type channels (Jakoubek et al. 2006) and Rho-kinase-mediated $\mathrm{Ca}^{2+}$ sensitization (this study) are essential for HFPV.

The finding of HFPV prevention by the blockade of the Rho-kinase is further corroborated by our $\mathrm{P} / \mathrm{Q}$ ramp data. In general, the pressure values were lower for any given level of flow rate in the fasudil-treated group than in the controls during the hypoxic challenge. Furthermore, our data show that the difference was not due to a difference in the extrapolated pressure axis intercept. This parameter might be assumed to reflect critical closing pressure in the fetoplacental vascular bed. That, in turn, is determined by vascular tension in peripheral, collapsible vessels and their surrounding pressure. By contrast, the differences in $\mathrm{P} / \mathrm{Q}$ lines were due to a reduced slope in the fasudil-treated group as compared to controls. In lungs this parameter reflects vascular resistance upstream from the collapsible segment of the vascular bed (Herget and Hampl 1995). Thus, the site of the HFPV sensitive to Rho-kinase inhibition appears to be upstream from the most peripheral, collapsible vessels. However, the issue of exact localization of HFPV awaits further exploration.

Fetoplacental circulation is quite distinct from other vascular beds (and similar to the adult pulmonary vasculature) because under normal conditions it has minimal or no resting vascular tone. This can be demonstrated by the absence of a response to a large dose of a strong vasodilator such as sodium nitroprusside (Hampl et al. 2002). It is interesting, therefore, that in the present study fasudil was able to reduce significantly the resting vascular resistance. Similarly in lungs Rho-kinase inhibitor Y-27632 caused a reduction of the baseline perfusion pressure (Nagaoka et al. 2004). It suggests that fasudil is even more potent vasodilator than sodium nitroprusside.

The full recovery of the fasudil-blocked HFPV by the replacement of the fasudil-containing perfusate with a fresh one without fasudil indicates that the inhibition of HFPV by fasudil was not due to edema or some other artifact caused by the prolonged artificial perfusion of the preparation.

In conclusion, the present study shows that Rhokinase-mediated $\mathrm{Ca}^{2+}$ sensitization contributes to the maintenance of resting fetoplacental vascular resistance and plays a key role in the mechanism of HFPV. Rhokinase inhibitors thus could be a potentially useful therapeutic instrument for diseases such as intrauterine growth restriction, where HFPV is considered a key pathophysiological factor.

\section{Conflict of Interest}

There is no conflict of interest.

\section{Acknowledgements}

The study was supported by the Grant Agency of the Czech Republic 305/08/0108 and by the Second Faculty of Medicine, Charles University in Prague.

\section{References}

FAGAN KA, OKA M, BAUER NR, GEBB SA, IVY DD, MORRIS KG, MCMURTRY IF: Attenuation of acute hypoxic pulmonary vasoconstriction and hypoxic pulmonary hypertension in mice by inhibition of Rho-kinase. Am J Physiol 287: L656-L664, 2004.

FUKATA Y, AMANO M, KAIBUCHI K: Rho-Rho-kinase pathway in smooth muscle contraction and cytoskeletal reorganization of non-muscle cells. Trends Pharmacol Sci 22: 32-39, 2001.

HAMPL V, BIBOVA J, STRANAK Z, WU X, MICHELAKIS ED, HASHIMOTO K, ARCHER SL: Hypoxic fetoplacental vasoconstriction in humans is mediated by potassium channel inhibition. Am $J$ Physiol 283: H2440-H2449, 2002.

HERGET J, HAMPL V: Pulmonary circulation. In: Biomechanics of the Cardiovascular System. BB SRAMEK, J VALENTA, F KLIMES (eds), Czech Technical University Press, Praha, 1995, pp 327-336.

HOWARD RB: Control of human placental blood flow. Med Hypotheses 23: 51-58, 1987.

JAKOUBEK V, BIBOVA J, HAMPL V: Voltage-gated calcium channels mediate hypoxic vasoconstriction in the human placenta. Placenta 27: 1030-1033, 2006. 
LEACH RM, ROBERTSON TP, TWORT CH, WARD JP: Hypoxic vasoconstriction in rat pulmonary and mesenteric arteries. Am J Physiol 266: L223-L231, 1994.

NAGAOKA T, MORIO Y, CASANOVA N, BAUER N, GEBB S, MCMURTRY I, OKA M: Rho/Rho kinase signaling mediates increased basal pulmonary vascular tone in chronically hypoxic rats. Am J Physiol 287: L665-L672, 2004.

ROBERTSON TP, DIPP M, WARD JP, AARONSON PI, EVANS AM: Inhibition of sustained hypoxic vasoconstriction by Y-27632 in isolated intrapulmonary arteries and perfused lung of the rat. Br J Pharmacol 131: 5-9, 2000.

SOMLYO AP, SOMLYO AV: $\mathrm{Ca}^{2+}$ sensitivity of smooth muscle and nonmuscle myosin II: modulated by $\mathrm{G}$ proteins, kinases, and myosin phosphatase. Physiol Rev 83: 1325-1358, 2003.

STULC J, STULCOVA B: Transport of calcium by the placenta of the rat. J Physiol (Lond) 371: 1-16, 1986.

SYLVESTER JT, SHIMODA LA, AARONSON PI, WARD JPT: Hypoxic pulmonary vasoconstriction. Physiol Rev 92: 367-520, 2012.

WANG Z, JIN N, GANGULI S, SWARTZ DR, LI L, RHOADES RA: Rho-kinase activation is involved in hypoxiainduced pulmonary vasoconstriction. Am J Respir Cell Mol Biol 25: 628-635, 2001. 